\title{
CMS inner detector: the Run 1 to Run 2 transition and first experience of Run 2
}

\section{Clemens Lange, on behalf of the CMS Collaboration*}

Physik-Institut, Universität Zürich, Winterthurerstrasse 190, 8057 Zurich, Switzerland

E-mail: clemens.lange@cern.ch

The CMS inner detector is the largest silicon tracker ever built. Located around the center of CMS where the LHC particle beams are brought into collision, it consists of a hybrid pixel detector with 66 million channels and a $200 \mathrm{~m}^{2}$ silicon strip detector with about 10 million readout channels. The tracking detector provides high-resolution measurements of charged particles passing through a 3.8 T magnetic field and is also crucial for vertex reconstruction. This report briefly summarises the operational experience gained with this detector during the first three years of LHC operation (Run 1) including performance measurements. The focus lies on the work carried out during the first long shutdown of LHC to prepare the detector for the high-radiation and high-luminosity environment of Run 2. The most important tasks were the repair of the detector and preparations for operating the detector at significantly lower temperature. First experience with 2015 operation of the detector is also presented.

24th International Workshop on Vertex Detectors

1-5 June 2015

Santa Fe, New Mexico, USA

${ }^{*}$ Speaker. 


\section{Introduction}

The CMS detector encloses the interaction region located at point 5 of the Large Hadron Collider (LHC) ring at CERN and is one of two general purpose experiments constructed to discover the Higgs boson and to search for new physics at high energies [1]. The LHC was initially designed to collide bunches of $1.2 \times 10^{11}$ protons every $25 \mathrm{~ns}$ at a centre-of-mass energy of $14 \mathrm{TeV}$ for an expected instantaneous luminosity of $10^{34} \mathrm{~cm}^{-2} \mathrm{~s}^{-1}$. The CMS detector was consequently constructed to operate in an environment with this high rate of interactions, maintaining high efficiency and excellent resolution as well as good particle identification. To accomplish this, the detector is designed in an onion-like structure that comprises several sub-systems: The innermost part of the detector consists of the pixel detector, and the strip tracker, which are the two tracking systems of CMS. Surrounding them, calorimeter systems are placed that measure the energy of the particles created in the collision and subsequent decays. All these detectors are submerged in the central feature of CMS: a $3.8 \mathrm{~T}$ superconducting solenoid of $6 \mathrm{~m}$ internal diameter. The outermost layer of the detector, the muon system, is installed between the layers of the steel flux-return yoke of the solenoid.

This report focusses on the CMS pixel and strip tracker detectors, specifically on details relating to the operation of the detector during the first years of LHC running from 2010 to 2012 (Run 1). The technologies employed are described, along with the properties of the detectors and their performance during Run 1. Special attention is given to work during the long shutdown of the LHC until early 2015. First experience with the repaired and improved detectors and their environment in 2015 is presented.

\section{The CMS pixel and silicon strip detectors}

The CMS pixel detector is a semiconductor tracker consisting of three barrel layers (BPix) at radii of 4.3, 7.2, and $11 \mathrm{~cm}$ from the centre of the detector, and two endcap pixel disks at each side (FPix). The layers are composed of modular detector units that consist of thin segmented silicon sensors. The sensor material is an $n^{+}$implant in $n$-type bulk and each pixel has dimensions of $100 \times 150 \mu \mathrm{m}^{2}$ with a $250 \mu \mathrm{m}$ sensor thickness. The pixels are grouped in arrays of $52 \times 80$ and bump-bonded to a readout chip (ROC) [2]. Sixteen ROCs make up a full module. The ROCs are wire-bonded to the high density interconnect (HDI), which is a low-mass printed circuit board. On this HDI the Token Bit Manager (TBM) is located, which distributes clock and trigger to the ROCs, and manages the ROC controls and the analogue readout [3]. The analogue electrical signal is transduced to an optical one by so-called analogue optical hybrids (AOHs) and then transmitted to the front end drivers (FEDs) via optical fibres for digitisation and distribution to the central CMS data acquisition (DAQ) system. Sets of eight modules are screwed on thin carbon fibre ladders that are glued to aluminium cooling pipes. Ladders are mounted on the layer half-shells, of which three make up half of the barrel detector. Each half is connected to two supply tube half-cylinders (one to the left and one to the right) containing the level translator chips as well as analogue- and digitaloptohybrid circuits. In addition, each supply tube provides the power and cooling lines to a quarter of the detector. The forward pixel disks are also divided into two halfs with a turbine-like geometry to enhance charge sharing. In total, the barrel pixel detector contains 48 million pixels making up 
an area of $0.78 \mathrm{~m}^{2}$ while the endcap disks contain 18 million pixels of an area of $0.28 \mathrm{~m}^{2}$ adding up to about 66 million channels.

The CMS silicon strip tracker completes the CMS tracking system, which in total provides high precision measurements of charged particle trajectories up to an absolute pseudo-rapidity value of $|\eta|<2.5^{1}$. It consists of ten layers in the barrel region, four in the inner (TIB), and six in the outer barrel (TOB). The forward region is complemented by three rings of inner disks (TID), and nine rings of outer disks (TEC). In the outer parts of the silicon tracker-TOB and TEC rings 5 to 7-two silicon sensors are daisy-chained to form the silicon module. In four layers in the barrel region and three rings of the endcap region, the strip tracker uses so-called stereo modules. For these, two silicon sensors are mounted back-to-back with a $100 \mathrm{mrad}$ stereo angle to effectively provide a 3D hit resolution. The sensor material is $p^{+}$-in- $n$ silicon, which has a thickness of 320 and $500 \mu \mathrm{m}$ for the inner and outer parts of the detector, respectively. The strip pitch ranges from 83 to $205 \mu \mathrm{m}$ and also the number of strips varies from 512 to 768 depending on the module location. All together, the strip tracker has a total active area of $200 \mathrm{~m}^{2}$ and about 9.6 million electronic channels. The readout of the detector is analogue and is performed via applicationspecific integrated circuits called APV25 [4]. To cool both tracking detectors, a mono-phase $\mathrm{C}_{6} \mathrm{~F}_{14}$ cooling system is used.

\section{Run 1 performance}

LHC provided proton-proton collisions from 2010 on, first at a centre-of-mass energy of $7 \mathrm{TeV}$ until the end of 2011, and then at $8 \mathrm{TeV}$ for the whole of 2012. There were also intermediate periods where heavy ion collisions took place, but these are not covered in details here. The machine reached a peak instantaneous luminosity of $7.7 \times 10^{33} \mathrm{~cm}^{-2} \mathrm{~s}^{-1}$, with up to 20 hard interactions per single bunch crossing on average. By the end of Run 1, LHC delivered $6 \mathrm{fb}^{-1}$ of data at $7 \mathrm{TeV}$ and $23 \mathrm{fb}^{-1}$ of data at $8 \mathrm{TeV}$. LHC operation stopped in early 2013 to upgrade the machine during a first long shutdown period (LS1) until early 2015.

The tracking detectors both performed according to expectation. The pixel detector showed an overall hit efficiency above $99 \%$ for all working ROCs. However, a dynamic inefficiency was observed with increasing instantaneous luminosity, which is due to the fact that a higher occupancy increases probability of ROC buffer overflow. The measured $r-\phi$ resolution ${ }^{2}$ was below $10 \mu \mathrm{m}$. Hit efficiency and resolution are shown in Fig. 1. The strip tracker performed equally well with a hit efficiency considering all modules of around $92 \%$ and above $99 \%$ if only considering working modules. The hit resolution was measured to values of 16 to $42 \mu \mathrm{m}$ depending on the strip pitch of the modules.

At the end of Run 1, about $2.3 \%$ of all channels in the barrel pixel detector were not operational anymore. The main reasons were broken wire-bond connections between the ROC and the HDI as well as issues with the laser and the AOH. Furthermore, some modules had an old ROC design,

\footnotetext{
${ }^{1} \mathrm{CMS}$ uses a right-handed coordinate system with the $z$-axis pointing along the LHC counter-clockwise beam direction, the $x$-axis pointing toward the center of the ring, and the $y$-axis pointing upward. The pseudo-rapidity is defined as $\eta=-\ln \tan (\theta / 2)$, where $\theta$ is the polar angle.

${ }^{2}$ The azimuthal angle, $\phi$, is measured in the $x-y$ plane relative to the $x$-axis, the radial coordinate in this plane is denoted by $r$.
} 

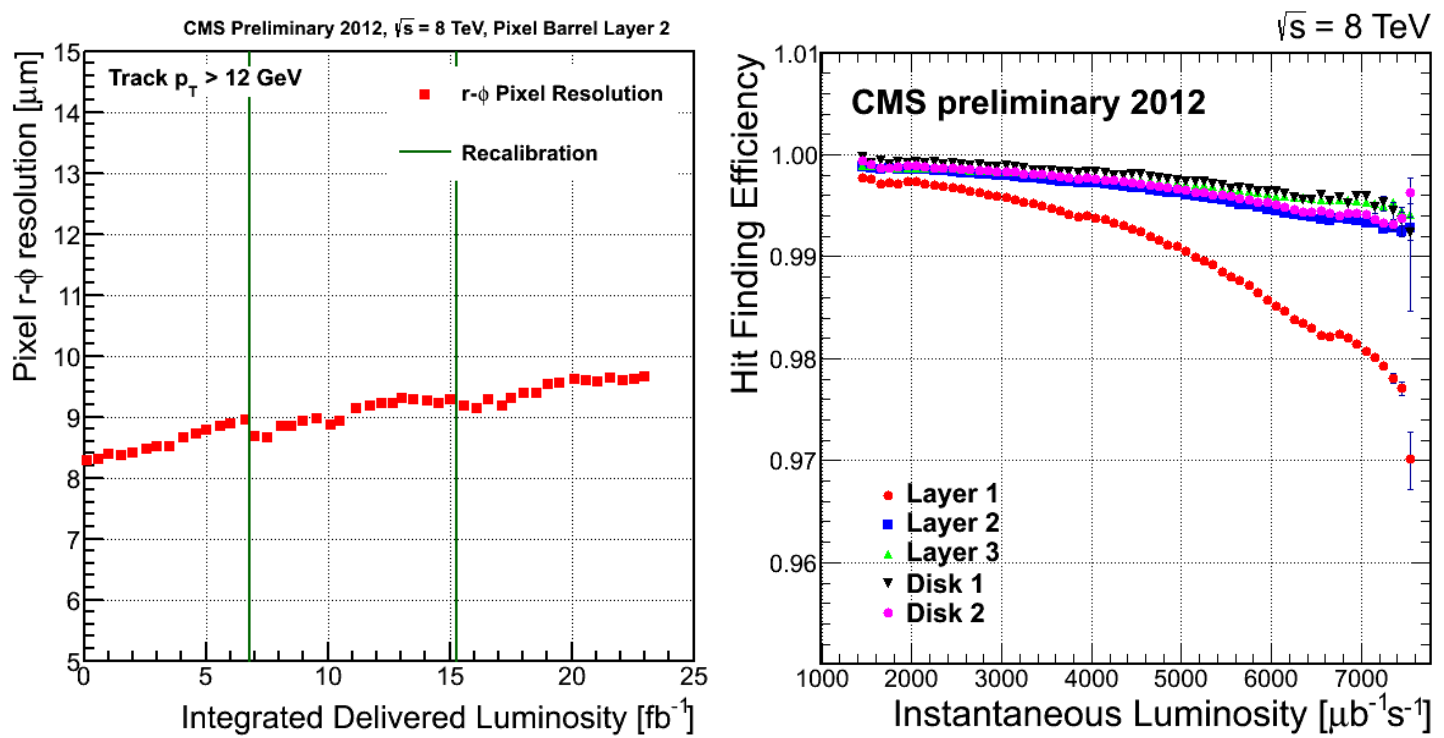

Figure 1: Resolution of the pixel detector in $r-\phi$ as a function of integrated luminosity (left), and hit finding efficiency as a function of instantaneous luminosity (right) for LHC Run 1 [5].

which caused operational problems, and were therefore disabled. For the pixel endcap disks, 7.8\% of the detector were non-operational. The main reason were channels that were masked since they had a long rise time in the analogue readout leading to pixel address misreadings and consequently a miscounting of ROCs and events. Another reason for disabling channels were broken optical readouts. For the strip tracker $2.4 \%$ of all channels were inactive at the end of 2012. Here, the main reasons among other less frequent ones were shorts of control rings, which are used for the communication between the front-end controller and the control and communication units, or missing control rings, shorts or open high voltage lines as well as issues with the optical fibres.

\section{Work during LHC long shutdown 1}

The first long shutdown of the LHC was used to repair the pixel detector on the surface. It was extracted from CMS in summer 2013 and kept in cooling boxes in a cleanroom at the point 5 experimental area. For FPix, almost all disabled channels were repaired so that it was 99.9\% operational. The issues that were fixed were misaligned flex cables that made up $46 \%$ of the faulty channels, unplugged analogue electrical-to-optical converters (AOHs) making up $40 \%$ of the faulty channels, and generally problematic modules that were replaced (remaining $14 \%$ of faulty channels). For BPix, replacements were only attempted for the layer 3 outer shell, which made up $52 \%$ of the faulty channels, since the other layers and the inner shell of layer 3 were considered too risky to touch without breaking further parts. Two additional AOHs were replaced that had until then been operated using a backup connection. In the end, the barrel pixel detector was about $99 \%$ operational again.

There was, however, a serious incident in mid-August 2014. After having replaced a BPix module, tests of the corresponding quadrant showed severe damage: 55 modules were found to be not working anymore. It was decided to take that part of the detector to Paul Scherrer Institute 
(PSI), Switzerland, where it had originally been constructed, for further tests and repairs. Shorts were discovered at the ROCs between two pins, which were mostly close to the SMD components. Furthermore, several modules also showed multiple shorts between the TBM pads and the cable pads. Eventually, the detector was repaired using 40 new modules and 19 repaired ones within three months time, which was only possible thanks to the availability of spare components, expertise, and good manufacturer relations. The shorts were suspected to be caused by humidity due to unobserved condensation in the cooling box. In particular, problems were found for modules that had a common pad passivation window with small trenches between the pins where solder flux residue and later humidity could have accumulated. For modules with individual pad passivation window that in particular have walls between the pins, this was not observed. Photos and a sketch of the problem analysis are shown in Fig. 2.
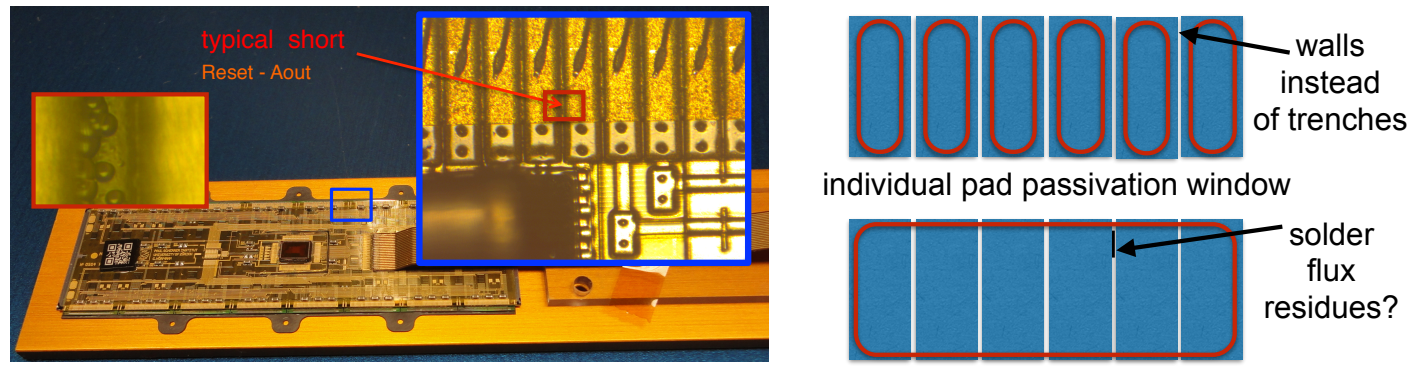

individual pad passivation window

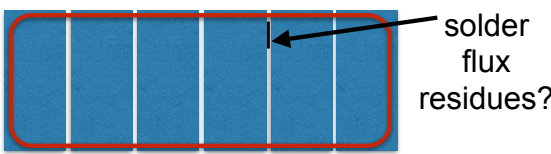

common pad passivation window

Figure 2: Photos (courtesy of PSI) of a typical short observed between two ROC pins (left) and sketch of the difference between the two pad passivation methods.

Another major effort during the long shutdown was to improve the tracker cooling system. Originally, the tracking detectors were designed to operate at low temperatures, in particular at $-20^{\circ} \mathrm{C}$ for the pixel detector, to reduce sensor leakage currents, which are reduced by about $50 \%$ every $7 \mathrm{~K}$. Furthermore, this would prevent reverse annealing, which would eventually require too high depletion voltages. For Run 1, the dew points in the tracker bulkhead and the service channels - which are the end point and path, respectively, of all tracker services like power, cooling, and dry gas-were not sufficiently low, mainly due to imperfect sealing. Therefore, LS1 was used to improve the sealing, insulation and also to upgrade the $\mathrm{C}_{6} \mathrm{~F}_{14}$ cooling and dry gas plants to run the detectors at their design temperatures. Nevertheless, in order to avoid pushing the cooling system to its limits, the decision was taken to operate the pixel detector at $-10^{\circ} \mathrm{C}$ and the strip tracker at $-15^{\circ} \mathrm{C}$ until the next long shutdown, which is currently planned for 2018. At that point, the detector is expected to have seen an integrated luminosity of about $150 \mathrm{fb}^{-1}$, and the cooling will again be improved to stably operate at $-20^{\circ} \mathrm{C}$. The figure of merit for this decision was the required depletion voltage for the strip tracker at its end-of-life time in $2023\left(500 \mathrm{fb}^{-1}\right)$, which is only increased by less than $15 \mathrm{~V}$ when running at $-15^{\circ} \mathrm{C}$ as opposed to $-20^{\circ} \mathrm{C}$ until 2018 , as shown in Fig. 3 (left).

\section{Re-commissioning of the detectors}

The barrel pixel detector was installed back into CMS on $8^{\text {th }}$ December 2014, three months 

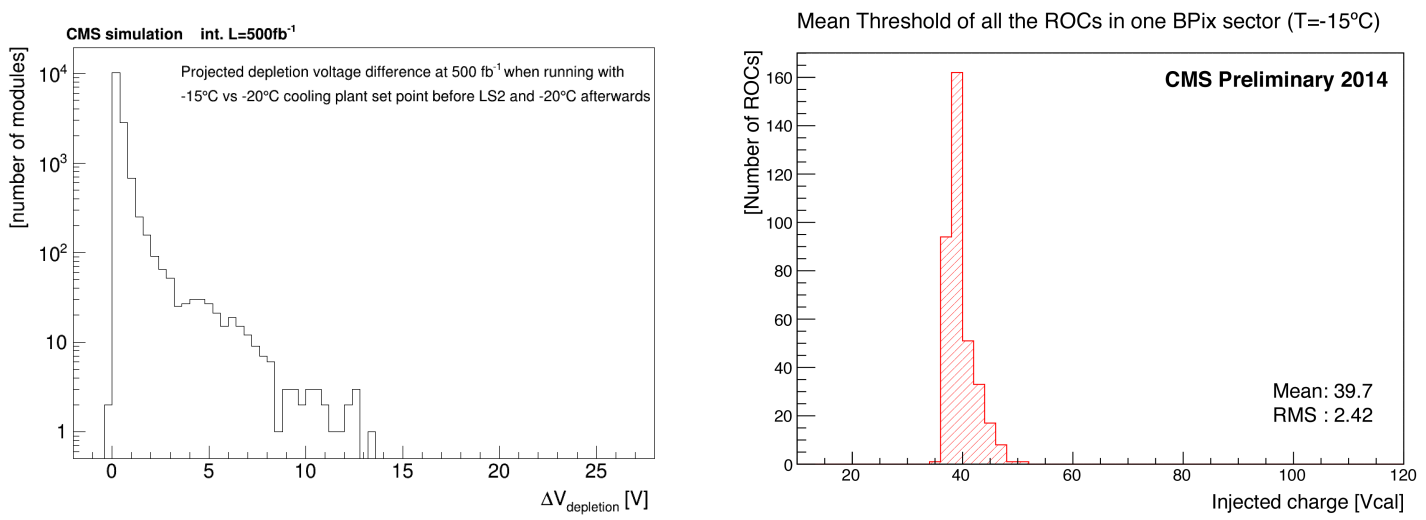

Figure 3: Simulated difference of full depletion voltage for the strip tracker at $500 \mathrm{fb}^{-1}$ when running between LS1 and LS2 $\left(\approx 150 \mathrm{fb}^{-1}\right)$ at $-15^{\circ} \mathrm{C}$ and afterwards at $-20^{\circ} \mathrm{C}$ until LS3 instead of running at $-20^{\circ} \mathrm{C}$ for the whole time [6] (left), and distribution of the minimised thresholds for all the ROCs in one BPix sector at $-15^{\circ} \mathrm{C}$ measured in the cleanroom [5] (right).

later than originally planned due to the incident mentioned above. Particular care was given to the centring of the detector with respect to the new beampipe, required for the pixel phase 1 upgrade planned for 2016-2017, since before it had been slightly shifted irradiating one side stronger than the other. The following day, all cooling loops, power cables and fibres were connected, and first attempts to power the detector and to test a sector were made. The detector was then checked out over the course of the next three days at room temperature of about $16^{\circ} \mathrm{C}$. The FPix detector was installed on $13^{\text {th }}$ December 2014 following a similar check-out procedure as for BPix so that most work was already completed before Christmas. The third and outermost FPix disk that had so far been empty was equipped with a pilot system for the Phase 1 upgrade of the pixel detector [7, 8].

The full pixel detector was re-commissioned in January 2015 within about a fortnight using a procedure that had been exercised and improved during LS1. An example calibration performed in the cleanroom is shown in Fig. 3 (right). The basic set of calibrations was run including the analogue voltage adjustment for the ROCs, the pixel comparator threshold calibration as well as the pulse height optimisation and a gain calibration. Furthermore, the switch to the new CMSwide trigger and timing system was successfully completed. The tracker bulkheads were closed on $29^{\text {th }}$ January to end LS1. In total, BPix had about $1 \%$ and FPix $0.04 \%$ dead or disabled channels.

For the strip tracker, most of the work during LS1 went into establishing the cooling system. No repairs of the detector were performed so that $97.6 \%$ of the modules were operational at the beginning of LHC Run 2. New commissioning software as well as a new computing infrastructure and several software and firmware upgrades aided the commissioning process to obtain a smoothly operating detector.

\section{Start of 2015 data-taking}

Global CMS data-taking commenced with a run called CRUZET (Cosmic RUn at ZEro Tesla), which started on $10^{\text {th }}$ February. This allowed scans to find the optimal timing both internally and also with respect to the whole of CMS. Furthermore, a small fraction $(<0.1 \%)$ of pixels that 
were found to be noisy were masked. A total of 420 hours of cosmic data were recorded. The second commissioning run called CRAFT (Cosmic Run At Four Tesla) began on $19^{\text {th }}$ March during which about 3 million cosmic events were taken resulting in approximately 1.8 million tracks. On $20^{\text {th }}$ May first LHC test collisions at the unprecedented centre-of-mass energy of $13 \mathrm{TeV}$ took place during which the strip tracker took data. The pixel detector remained off to not risk potential damage to it since the proton beams had not been declared as stable by the LHC operators.

\section{Summary}

The CMS tracking detectors, the pixel detector and the strip tracker, have been running successfully during LHC Run 1. During the first long shutdown of the LHC, the pixel detector was repaired to about $99 \%$ operationality, the cooling of the tracking detectors has been significantly improved and several other improvements were put into place. After re-commissioning the detectors, it was already shown that they operate as expected again, and they are now ready to face the challenges of LHC Run 2.

\section{References}

[1] S. Chatrchyan et al. [CMS Collaboration], "The CMS experiment at the CERN LHC," JINST 3 S08004 (2008).

[2] H. C. Kästli et al., "Design and performance of the CMS pixel detector readout chip," Nucl. Instrum. Meth. A 565188 (2006) [physics/0511166].

[3] D. Kotlinski et al., "The control and readout systems of the CMS pixel barrel detector," Nucl. Instrum. Meth. A 56573 (2006).

[4] M. J. French et al., "Design and results from the APV25, a deep sub-micron CMOS front-end chip for the CMS tracker," Nucl. Instrum. Meth. A 466, 359 (2001).

[5] The CMS Tracker Collaboration, "Pixel performance plots," https://twiki.cern.ch/twiki/bin/view/CMSPublic/PixelOfflinePlots2013

[6] The CMS Tracker Collaboration, "Tracker commissioning plots," https://twiki.cern.ch/twiki/bin/view/CMSPublic/StripTrackerCommissioningPlots2014

[7] B. Akgün, "Pilot System for the Phase 1 Pixel Upgrade," these proceedings.

[8] L. Caminada, "The phase-1 upgrade of the CMS vertex detector," these proceedings. 